\title{
Serum metabolomic profile and potential biomarkers for severity of fibrosis in nonalcoholic fatty liver disease
}

\author{
Katsutoshi Tokushige $\cdot$ Etsuko Hashimoto $\cdot$ Kazuhisa Kodama $\cdot$ Maki Tobari \\ Noriko Matsushita - Tomomi Kogiso - Makiko Taniai - Nobuyuki Torii • \\ Keiko Shiratori · Yasuhiro Nishizaki · Takushi Ohga · Yoshiaki Ohashi · \\ Takaya Sato
}

Received: 5 December 2012/ Accepted: 31 January 2013/Published online: 13 March 2013

(C) The Author(s) 2013. This article is published with open access at Springerlink.com

\begin{abstract}
Background Biomarker for usefulness in diagnosing advanced fibrosis in nonalcoholic fatty liver disease (NAFLD) is expected. In order to discover novel biomarkers for NAFLD and its pathogenesis, we performed matabolomics screening. Methods (1) The initial cohort was 44 NAFLD patients. (2) This validation cohort was 105 NAFLD patients, 26 primary biliary cirrhosis (PBC) patients, and 48 healthy controls. Using capillary electrophoresis and liquid chromatography with mass spectrometry, we analyzed low molecular weight metabolites in these groups.

Results 1. In the initial cohort, we found 28 metabolites associated with advanced fibrosis. Among them, 4 sulfated steroids showed the greatest difference. A decrease of dehydroepiandrosterone sulfate (DHEA-S) and $5 \alpha$-andro$\operatorname{stan}-3 \beta$ ol-17-one sulfate (etiocholanolone-S) was observed with the progression of fibrosis. Furthermore, 16 hydroxydehydroepiandrosterone sulfate (16-OH-DHEA-S)
\end{abstract}

Electronic supplementary material The online version of this article (doi:10.1007/s00535-013-0766-5) contains supplementary material, which is available to authorized users.

K. Tokushige $\cdot$ E. Hashimoto $(\bowtie) \cdot$ K. Kodama $\cdot$ M. Tobari

N. Matsushita $\cdot$ T. Kogiso $\cdot$ M. Taniai $\cdot$ N. Torii $\cdot$ K. Shiratori

Department of Internal Medicine and Gastroenterology,

Tokyo Women's Medical University, Tokyo, Japan

e-mail: drs-hashimoto@mti.biglobe.ne.jp

Y. Nishizaki

Tokyo Hospital, Tokai University, Tokyo, Japan

T. Ohga $\cdot$ Y. Ohashi

Human Metabolome Technology, Inc., Yamagata, Japan

T. Sato

Tsuruoka National College of Technology, Department

of Chemical and Biological Engineering, Tsuruoka, Japan increased with the progression of fibrosis. 2. In the validation cohort, the decrease of DHEA-S and etiocholanolone-S, as well as the increase of 16-OH-DHEA-S, with the progression of fibrosis was confirmed. The 16-OH-DHEAS/DHEA-S ratio and 16-OH-DHEA-S/etiocholanolone-S ratio were even more strongly associated with the grade of fibrosis. Among PBC patients, 16-OH-DHEA-S tended to be higher in stages 3 and 4 than in stages 1 and 2. However, levels of DHEA-S, etiocholanolone-S, and the two ratios were not associated with the stage of PBC.

Conclusion Several metabolic products were found to be biomarkers of fibrosis in NAFLD and could also be useful for diagnosis of this condition. Our findings suggested disturbance of hormone metabolism in NAFLD and might lead to the development of new therapy.

\section{Abbreviations \\ NAFLD Nonalcoholic fatty liver disease \\ PBC Primary biliary cirrhosis \\ DHEA-S Dehydroepiandrosterone sulfate \\ Etiocholanolone-S $5 \alpha$-androstan-3 $\beta$ ol-17-one sulfate \\ 16-OH-DHEA-S 16 hydroxydehydroepiandrosterone sulfate}

\section{Introduction}

Nonalcoholic fatty liver disease (NAFLD) has become the most common type of liver disease in developed countries. NAFLD covers a wide spectrum from simple steatosis to nonalcoholic steatohepatitis (NASH), which can progress to cirrhosis and hepatocellular carcinoma (HCC) [1-3]. NASH was initially identified as a clinicopathological entity, and biopsy is still considered to be the "gold standard" for definitive diagnosis. However, liver biopsy has 
several drawbacks because it is an invasive, painful, and costly procedure that is associated with the possibility of sampling error and variability in interpretation. Moreover, the extremely high prevalence of NAFLD in the general population makes liver biopsy unsuitable as a diagnostic procedure for NASH. These shortcomings and drawbacks of liver biopsy highlight the urgent need to find noninvasive markers, such as biochemical markers, or imaging techniques for the assessment of NASH. So far, biomarkers have been evaluated for usefulness in distinguishing NASH from NAFLD and/or diagnosing advanced fibrosis or cirrhosis [4].

Recently, Younossi et al. [5] reported that a number of pathologic features were associated with hepatic mortality on univariate analysis, but fibrosis was the only independent predictor according to multivariate analysis, so the severity of fibrosis rather than a diagnosis of NASH determined hepatic mortality. In other words, liver fibrosis is the most important index of the severity of NAFLD.

Metabolomics involves the measurement of a large number of low molecular weight metabolites, including sugars, amino acids, and hormones, and it has emerged as a powerful tool for discovering novel biomarkers. Metabolomics has already identified new biomarkers for prostate cancer [6], Parkinson's disease [7], type 2 diabetes [8], acute myocardial infarction [9], and preeclampsia [10]. This promising area of research has also recently provided some important insight into the pathogenesis of human NAFLD and NASH [11-13]. However, sample size was small or their method was not in detail. We have attempted to discover novel fibrosis markers by detail method and their pathological role in the progression of NAFLD.

The present study was performed to investigate the differences of serum metabolomics between NAFLD patients with or without advanced fibrosis and to validate any novel biomarkers identified by the initial metabolomic analysis in another cohort of subjects.

\section{Materials and methods}

\section{Patients}

A total of 149 patients with a clinical and/or histological diagnosis of NAFLD made at Tokyo Women's Medical University between 2008 and 2011 were enrolled in this study. Forty-four patients formed the in initial cohort and 105 patients were used as the validation cohort. Diagnosis of NAFLD was based on the following criteria: (1) macrovesicular steatosis affecting at least $5 \%$ of hepatocytes, (2) intake of less than $20 \mathrm{~g}$ of ethanol per day (confirmed by the attending physician and family members residing with the patient), and (3) appropriate exclusion of other liver diseases such as alcoholic liver disease, viral hepatitis, autoimmune hepatitis, drug-induced liver disease, primary biliary cirrhosis (PBC), and primary sclerosing cholangitis $[2,14]$.

A complete history was obtained and physical examination was performed in all patients, followed by measurement of the following laboratory parameters: aspartate aminotransferase (AST), alanine aminotransferase (ALT), platelet count, hepatitis B serology (hepatitis B surface antigen, antibody to hepatitis B surface antigen, and hepatitis B core antigen antibody), hepatitis C serology (hepatitis $\mathrm{C}$ virus antibody), and autoantibodies (antinuclear antibody and antimitochondrial antibody). All liver biopsy specimens were examined by using hematoxylin-eosin, Mallory, and silver reticulin staining. Fibrosis was scored on a 5-grade scale: F0, normal connective tissue; F1, focal perivenular or pericellular fibrosis in zone 3; F2, perivenular or pericellular fibrosis confined to zones 3 and 2 with portal/periportal fibrosis; F3, bridging or septal fibrosis; and F4, cirrhosis $[14,15]$. Steatosis was graded on a scale of 1 to $3: 1$, mild (affecting 5-33\% of hepatocytes); 2, moderate (33-66\% of hepatocytes); 3 , severe ( $>66 \%$ of hepatocytes). Inflammation was graded as mild, moderate or severe based on the pathologist's overall impression.

The healthy control subjects (HC) were volunteers. They were all Japanese and were matched for age with the NAFLD patients. All control subjects were confirmed to have normal liver function and no viral hepatitis.

Twenty-five PBC patients were used as the disease control group. The diagnosis of $\mathrm{PBC}$ was based on the following three criteria: (1) detection of serum anti-mitochondrial antibody, (2) elevated liver enzymes at the initial diagnosis, and (3) histological features compatible with PBC on liver biopsy [16]. Fibrosis was classified according to Scheuer's classification [17] and the PBC patients were divided into 2 groups (stage 1-2 or stage 3-4). PBC patients had no other chronic liver diseases, including viral hepatitis.

Informed consent was obtained from all subjects before enrollment in the study. The study protocol conformed to the ethical guidelines of the 2008 Declaration of Helsinki and was approved by our institution's research committee.

\section{Sample collection and metabolomics analysis}

Serum was obtained by centrifugation of blood samples for $10 \mathrm{~min}$ at $1500 \times \mathrm{g}$ and $4{ }^{\circ} \mathrm{C}$, and was stored at $-80{ }^{\circ} \mathrm{C}$. Preparation of serum for capillary electrophoresis (CE)TOF mass spectrometry (MS) or liquid chromatography (LC)-TOFMS and measurement were performed as described previously [18]. With each system, two mode of the measurement were employed to detect both of cationic and anionic metabolites. Identification of compounds from the peaks was based on the annotated tables of $\mathrm{m} / \mathrm{z}$ values and normalized migration times. 
Important metabolites were confirmed by measurement of standard compounds. As chemical standards, dehydroepiandrosterone sulfate (DHEA-S) and $5 \beta$-androstan- $3 \alpha$ ol-17-one sulfate (etiocholanolone-S) were purchased from Sigma-Aldrich Corporation (St. Louis, MO, USA), 16 hydroxydehydroepiandrosterone sulfate (16-OH-DHEA-S) was purchased from Steraloids Inc. (Newport, RI, USA), and dehydroepiandrosterone disulfate (DHEA-2S) was obtained by the sulfation of dehydroepiandrosterone as reported previously [19].

\section{Identification of candidate biomarkers}

Initial cohort

The initial cohort was studied to seek candidate biomarkers for detection of advanced fibrosis. The 44 NAFLD patients in initial cohort were divided into 2 groups based on the severity of fibrosis (F0-2 vs. F3-4). We compared the two groups with respect to the results of metabolomics analysis. Peaks showing significant differences between the two groups were selected as potential predictors of advanced fibrosis.
Validation cohort

Several important biomarkers and their ratios were validated in another 105 NAFLD patients, 48 control subjects, and 25 PBC patients (disease control group). Further analysis was done by comparing NAFLD patients with control subjects or stage 1-2 versus stage 3-4 PBC patients.

Diagnosis of fibrosis

We investigated the diagnosis of fibrosis $\geq F 3$. All NAFLD patients from the combined initial and validation cohort were used for this study. Diagnostic performance was assessed by ROC analysis, with calculation of the area under the ROC curve (AUC).

Statistical analysis

Peak areas were normalized against those of the internal standards in each measurement mode and the resulting values (relative areas) were used for calculation. For assessment of the selected biomarkers, the absolute serum concentration was determined from the calibration

Table 1 Profile of the subjects

\begin{tabular}{|c|c|c|c|c|c|}
\hline A. Initial cohort & & & & & \\
\hline NAFLD & & & & & \\
\hline No. & 44 & & & & \\
\hline Age & $55.8 \pm 14.3(29-82)$ & & & & \\
\hline Gender $(\mathrm{M} / \mathrm{F})$ & $24 / 20$ & & & & \\
\hline Fibrosis grade & & Inflammation & & Steatosis & \\
\hline $0-1$ & 11 & $0-1$ & 8 & 1 & 11 \\
\hline 2 & 17 & 2 & 26 & 2 & 13 \\
\hline 3 & 10 & 3 & 10 & 3 & 20 \\
\hline 4 & 6 & & & & \\
\hline B. Validation cohs & & & & & \\
\hline NAFLD & & & & & \\
\hline No & 105 & & & & \\
\hline Age & $51.5 \pm 15.4(18-84)$ & & & & \\
\hline Gender $(\mathrm{M} / \mathrm{F})$ & $57 / 48$ & & & & \\
\hline Fibrosis grade & & Inflammation & & Steatosis & \\
\hline $0-1$ & 38 & $0-1$ & 26 & 1 & 28 \\
\hline 2 & 39 & 2 & 67 & 2 & 27 \\
\hline 3 & 14 & 3 & 12 & 3 & 50 \\
\hline 4 & 14 & & & & \\
\hline $\mathrm{PBC}$ & & & & & \\
\hline No. & 26 (Stage $1-2,20 ;$ Stage $3-4,6)$ & & & & \\
\hline Age & $64.2 \pm 12.8(47-90)$ & & & & \\
\hline Gender $(\mathrm{M} / \mathrm{F})$ & $1 / 25$ & & & & \\
\hline Healthy controls & & & & & \\
\hline No. & 48 & & & & \\
\hline Age & $55.7 \pm 3.4(50-60)$ & & & & \\
\hline Gender $(\mathrm{M} / \mathrm{F})$ & $35 / 13$ & & & & \\
\hline
\end{tabular}


curve obtained with standard chemicals added to serum. For the assessment of ratios between two markers, ratio values were changed into log-values. For comparison between groups of different stages, Steel-Dwass test for NAFLD or Welch's $t$ test for PBC was employed. The association between age and several metabolomic parameters was determined by Pearson's correlation coefficient analysis and correlation coefficients ( $R$ values) were calculated.

\section{Results}

Initial cohort

Forty-four NAFLD patients were analyzed and Table 1 shows their clinical data. The mean age was 55.8 years (range: $29-82$ years) and the male/female ratio was $24 / 20$. The fibrosis grade was F0-1 in 11 cases, F2 in 17 cases, F3 in 10 cases, and F4 in 6 cases. In our metabolomics analysis, 293 peaks were detected by the four modes of measurement and their areas were calculated. When we investigated the association between fibrosis and these peaks, 28 peaks showed significant differences between the F0-2 and F3-4 groups. Thus, these were candidate biomarkers for predicting advanced fibrosis (Table 2). Ten candidate markers were fatty acid components, 4 were steroid hormones, 2 were amino acids, 4 were amino acid metabolites, 2 were peptides, 2 were fatty metabolites, and 4 were other compounds. Four sulfated steroids showed the greatest difference between F0-2 and F3-4. Among them, etiocholanolone-S, DHEA-2S, and DHEA-S decreased with the progression of fibrosis, while 16-OH-DHEA-S increased with the progression of fibrosis.

Table 2 Potential biomarkers found by screening

\begin{tabular}{|c|c|c|c|c|}
\hline \multirow[t]{2}{*}{ ID } & \multirow[t]{2}{*}{ Annotation } & \multicolumn{3}{|l|}{ Relative area } \\
\hline & & $F<3$ & $F \geq 3$ & $p$ value \\
\hline NA03_513 & Etiocholanolone-S & $0.1022 \pm 0.0882$ & $0.0269 \pm 0.0269$ & 0.000 \\
\hline NA03_504 ${ }^{\mathrm{a}}$ & Nervonic acid & $0.0083 \pm 0.0027$ & $0.0144 \pm 0.0144$ & 0.002 \\
\hline NA03_703 & 16OHDHEA-S & $0.0182 \pm 0.0147$ & $0.0402 \pm 0.0402$ & 0.004 \\
\hline NA03_118 & Gln & $4.0088 \pm 1.3949$ & $5.0026 \pm 5.0026$ & 0.004 \\
\hline NA03_273 & Carnosine & $0.0036 \pm 0.0021$ & $0.0068 \pm 0.0068$ & 0.005 \\
\hline NA03_126 & Triethanolamine & $0.0035 \pm 0.0017$ & $0.0024 \pm 0.0024$ & 0.006 \\
\hline NA03_247 & Kynureine & $0.0207 \pm 0.0049$ & $0.0254 \pm 0.0254$ & 0.007 \\
\hline NA03_266 & DHEA-2S & $0.0363 \pm 0.0364$ & $0.0150 \pm 0.0150$ & 0.008 \\
\hline NA03_438 ${ }^{\mathrm{a}}$ & cis-11-Eicosenoic acid or cis-13-Eicosenoic acid & $0.0371 \pm 0.0228$ & $0.0585 \pm 0.0585$ & 0.014 \\
\hline NA03_036 & Hypotaurine & $0.0144 \pm 0.0053$ & $0.0107 \pm 0.0107$ & 0.018 \\
\hline NA03_141 ${ }^{\mathrm{a}}$ & Pelargonic acid & $0.0512 \pm 0.0089$ & $0.0450 \pm 0.0450$ & 0.019 \\
\hline NA03_239 & $O$-Acetylcarnitine & $0.3946 \pm 0.0806$ & $0.4802 \pm 0.4802$ & 0.020 \\
\hline NA03_183 & Serotonin & $0.0053 \pm 0.0025$ & $0.0037 \pm 0.0037$ & 0.020 \\
\hline NA03_021 ${ }^{\mathrm{a}}$ & Valeric acid & $0.0538 \pm 0.0391$ & $0.0352 \pm 0.0352$ & 0.022 \\
\hline NA03_170 & Decanoic aid & $0.0465 \pm 0.0108$ & $0.0403 \pm 0.0403$ & 0.026 \\
\hline NA03_087 & Gly-Gly & $0.0122 \pm 0.0080$ & $0.0207 \pm 0.0207$ & 0.027 \\
\hline NA03_497 & Acy1-carnitine (13:1) & $0.0121 \pm 0.0095$ & $0.0213 \pm 0.0213$ & 0.027 \\
\hline NA03_104 ${ }^{\mathrm{a}}$ & Octanoic acid & $0.0260 \pm 0.0064$ & $0.0226 \pm 0.0226$ & 0.030 \\
\hline NA03_121 & Glu & $2.3337 \pm 1.5646$ & $1.6048 \pm 1.6048$ & 0.033 \\
\hline NA03_236 & ADMA & $0.0087 \pm 0.0016$ & $0.0098 \pm 0.0098$ & 0.034 \\
\hline NA03_472 ${ }^{\mathrm{a}}$ & Erucic acid & $0.0037 \pm 0.0024$ & $0.0074 \pm 0.0074$ & 0.034 \\
\hline NA03_009 ${ }^{\mathrm{a}}$ & Butyric acid (or) isobutyric acid & $0.0341 \pm 0.0135$ & $0.0275 \pm 0.0275$ & 0.037 \\
\hline NA03_057 & Benzoic acid ${ }^{\mathrm{a}}$ & $0.0200 \pm 0.0032$ & $0.0183 \pm 0.0183$ & 0.038 \\
\hline NA03_ 102 & 1-Methyl-4-imidazoleacetic acid & $0.0064 \pm 0.0027$ & $0.0047 \pm 0.0047$ & 0.038 \\
\hline NA03_454 ${ }^{\mathrm{a}}$ & 15-HETE & $0.0128 \pm 0.0155$ & $0.0057 \pm 0.0057$ & 0.039 \\
\hline NA03_506 & DHEA-S & $0.2776 \pm 0.2319$ & $0.1424 \pm 0.1424$ & 0.041 \\
\hline NA03_069 & Pipecolic acid & $0.0497 \pm 0.0582$ & $0.0258 \pm 0.0258$ & 0.043 \\
\hline NA03_067 ${ }^{\mathrm{a}}$ & Heptanoic acid & $0.0188 \pm 0.0058$ & $0.0163 \pm 0.0163$ & 0.047 \\
\hline
\end{tabular}

${ }^{a}$ Fatty acid 


\section{Validation cohort}

Serum concentrations of three candidates were assessed in another 105 NAFLD patients, 25 PBC patients and $48 \mathrm{HC}$. A decrease of DHEA-S and etiocholanolone-S with the progression of fibrosis was confirmed (DHEA-S: HC, $3.06 \pm 1.38 ;$ NAFLD F0-1, $3.42 \pm 2.25 / \mathrm{F} 2,3.08 \pm 2.74 /$ F3, $2.18 \pm 1.91 / \mathrm{F} 4,0.66 \pm 0.74$; etiocholanolone-S: HC, $0.90 \pm 0.47 ;$ NAFLD F0-1, $0.91 \pm 0.80 / \mathrm{F} 2,0.68 \pm 0.92 /$ $\mathrm{F} 3,0.29 \pm 0.40 / \mathrm{F} 4,0.095 \pm 0.17$ ). An increase of 16-OHDHEA-S with the progression of fibrosis was also confirmed (HC, $0.15 \pm 0.07$; NAFLD F0-1, $0.26 \pm 0.22 / \mathrm{F} 2$, $0.41 \pm 0.42 / \mathrm{F} 3,0.64 \pm 0.52 / \mathrm{F} 4,0.49 \pm 0.41)(\mathrm{Fig} .1 \mathrm{a}-\mathrm{c})$.

The ratio of 16-OH-DHEA-S to DHEA-S (16/D) and the ratio of 16-OH-DHEA-S to etiocholanolone-S (16/E) were more clearly associated with the fibrosis grade (Fig. 1d-e) (16/D ratio: HC, $-1.31 \pm 0.18$; NAFLD F0-1,
$-1.15 \pm 0.47 / \mathrm{F} 2, \quad-0.86 \pm 0.50 / \mathrm{F} 3, \quad-0.43 \pm 0.48 / \mathrm{F} 4$, $-0.056 \pm 0.47 ; 16 / \mathrm{E}$ ratio: $\mathrm{HC},-0.76 \pm 0.25 ;$ NAFLD $\mathrm{F} 0-1,-0.46 \pm 0.76 / \mathrm{F} 2,-0.12 \pm 0.68 / \mathrm{F} 3,0.57 \pm 0.64 / \mathrm{F} 4$, $0.96 \pm 0.65)$.

NAFLD patients were divided by gender and separately compared the markers for mild fibrosis (F0-2) and severe fibrosis (F3-4) (supplementary Fig. 1A and B). In male cases $(n=57)$, all markers and both ratios were significantly different between mild fibrosis and severe fibrosis. In female cases $(n=48)$, etiocholanolone-S and both ratios were significantly different. DHEA-S and 16-OHDHEA-S were not significantly different, but showed similar tendencies.

Among the PBC patients, 16-OH-DHEA-S was higher in stages 3 and 4 than in stages 1 and $2(P=0.07)$. However, DHEA-S, etiocholanolone-S, and the two ratios were not associated with the stage of PBC (Fig. 2).

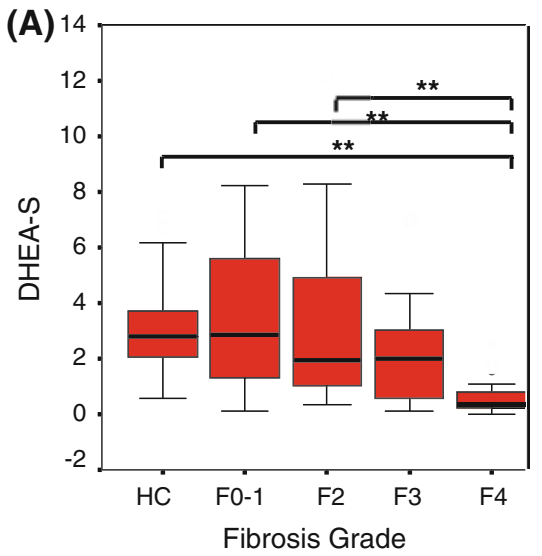

(B)
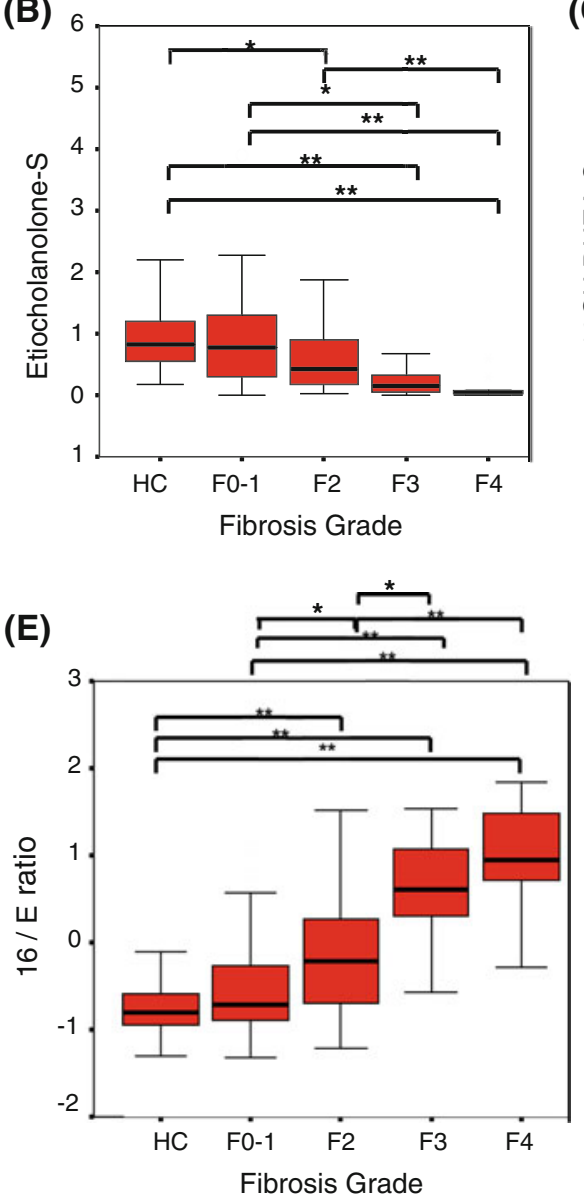

(C)

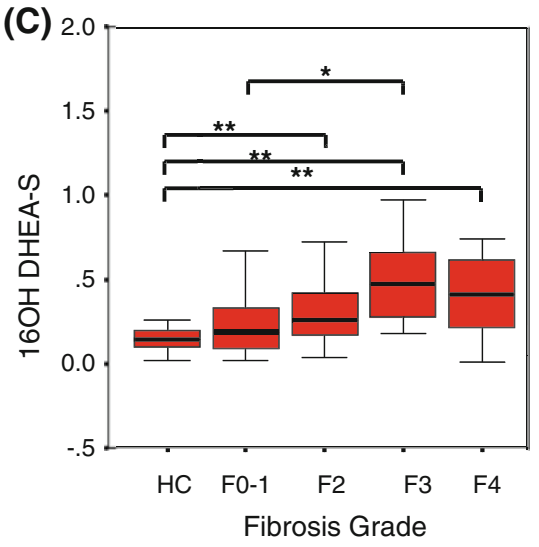

(D)

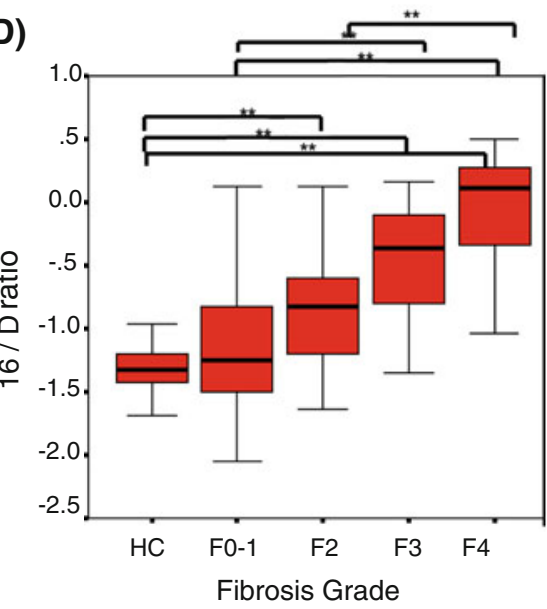

Fig. 1 Relationship between biomarkers and hepatic fibrosis grade. Serum concentrations of DHEA-S (a), etiocholanolone-S (b), and 16-OH-DHEA-S (c) in the NAFLD validation cohort. A decrease of DHEA-S and etiocholanolone-S, as well as an increase of $16-\mathrm{OH}-$ DHEA-S, were observed with the progression of fibrosis. Relationship between hepatic fibrosis grade and the 16-OH-DHEA-S/DHEA-S
(16/D) ratio or 16-OH-DHEA-S/etiocholanolone-S (16/E) ratio in the NAFLD validation cohort $(\mathbf{d}, \mathbf{e})$. For the assessment of ratios between two markers, ratio values were changed into log-values. Both ratios were clearly correlated with the fibrosis grade. $H C$ healthy control. $P P$ value by Steel-Dwass test 


\section{Diagnosis of fibrosis}

We investigated the diagnosis of fibrosis $\geq \mathrm{F} 3$ in 105 NAFLD patients of validation cohort (Fig. 3). Using ROC analysis, AUC, sensitivity and specificity for the three markers and two ratios were calculated. The AUC for DHEA-S, etiocholanolone-S, 16-OH-DHEA-S, the 16/D ratio, and the $16 / \mathrm{E}$ ratio was $0.74,0.81,0.74,0.85$, and 0.84 respectively.

\section{Discussion}

By metabolomics analysis, we found several biomarkers that were associated with progression of fibrosis in NAFLD, and our findings suggested disturbance of hormone metabolism in this disease.

In the present study, we investigated metabolites related to the severity of fibrosis in NAFLD. We identified 28 peaks with significant differences between patients with and without advanced fibrosis, which were candidate biomarkers. Most of these candidates are well known to have a role in the pathogenesis of NAFLD/NASH, including fatty acids, ADMA, DHEA-S, glutamate, carnosine, hypotaurine, serotonin, and pipecolic acid [11, 20-26]. However, other candidates have not been associated with the pathogenesis or progression of NAFLD.

We focused on sex hormone metabolites because of these marked differences. DHEA is a potential mediator of reactive oxygen species scavenger synthesis and has also been reported to augment insulin sensitivity [27]. DHEA-S is the most abundant circulating androgen, and it has already been reported to be useful for diagnosis and monitoring of fibrosis in NAFLD [21].

Etiocholanolone-S and 16-OH-DHEA-S were novel biomarkers detected in this study. Etiocholanolone- $S$ is one of the end products of androgen metabolism derived from testosterone (Fig. 4). Its biological significance in relation to liver disease is not clear. In the initial cohort, we also observed that DHEA-2S, which is another end product of androgen metabolism derived from DHEA-S, decreased along with the progression of fibrosis. In contrast, the serum level of 16-OH-DHEA-S, an intermediate of estrogen biosynthesis, was higher in patients with advanced fibrosis. This metabolite is DHEA-S with a hydroxyl group at position 16 and is formed during biosynthesis of estriol from DHEA in the fetal liver [28]. Although it has been reported that the serum level of 16-OH-DHEA-S was
Fig. 2 Relationship between biomarkers and PBC stage. Serum concentrations of DHEA-S (a), etiocholanolone-S (b), 16-OH-DHEA-S (c), and the two ratios $(\mathbf{d}-\mathbf{e})$ in $\mathrm{PBC}$ patients. 16-OH-DHEA-S was higher in stages 3 and 4 than in stages 1 and $2(P=0.07)$. However, DHEA-S, etiocholanolone-S, and the two ratios were not associated with the stage of PBC. NS not significant. $P P$ value by Welch's $t$ test. 16/D, 16-OH-DHEA-S/DHEA-S; 16/E, 16-OH-DHEA-S/ etiocholanolone-S

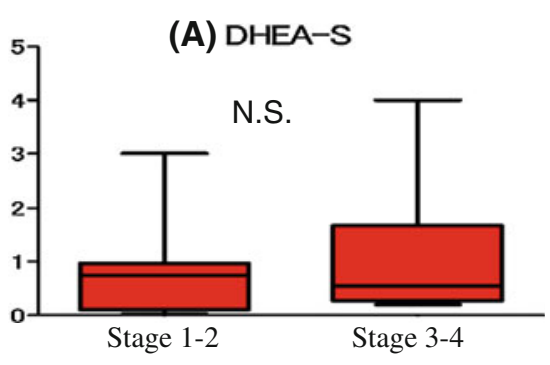

(B) Etiocholanolone-S

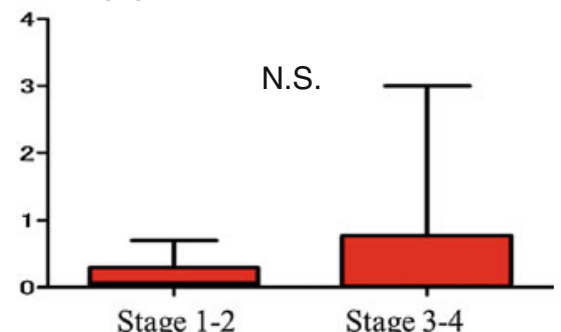

(D) $16 / \mathrm{D}$

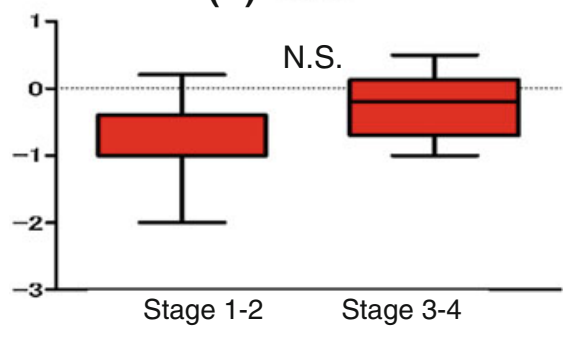

(C) $160 H$ DHEA-S

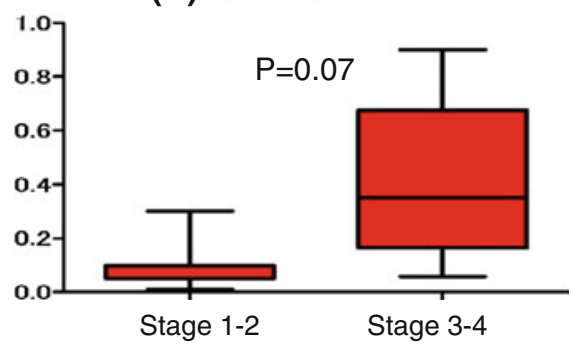

(E) $16 / E$

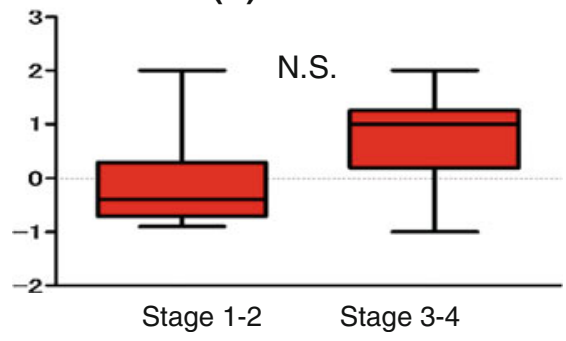


Fig. 3 Diagnosis of fibrosis $\geq$ F3 in 105 NAFLD patients of validation cohort. Using ROC analysis, AUC, sensitivity and specificity for the three markers and two ratios were calculated. 16/D, 16-OH-DHEA-S/DHEA-S; 16/E, 16-OH-DHEA-S/ etiocholanolone-S

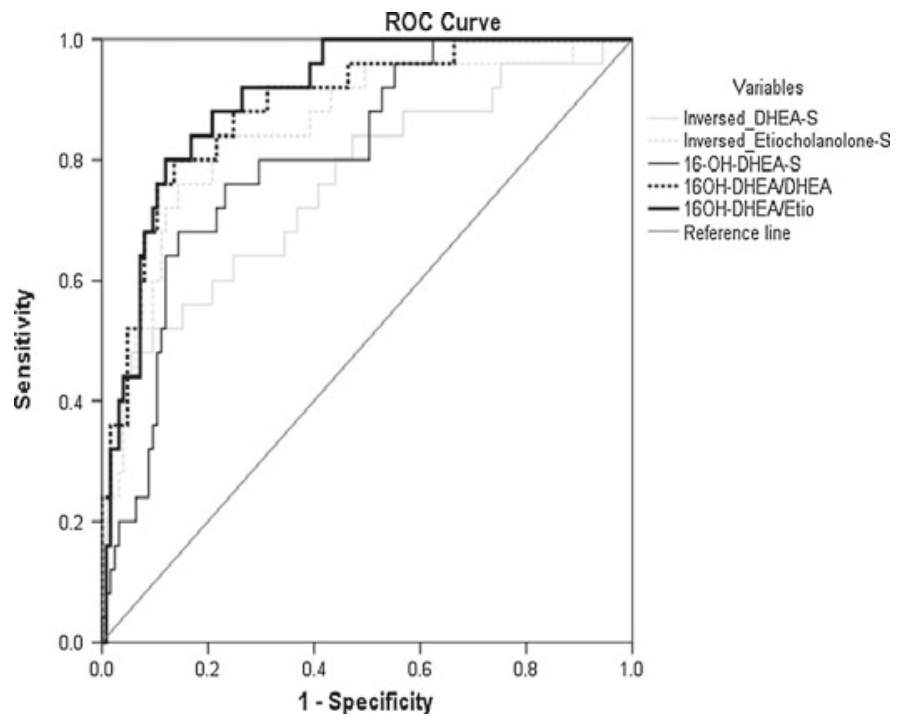

\begin{tabular}{|c|c|c|c|}
\hline & AUC & sensitivity & specificity \\
\hline DHEA-S & 0.74 & 61 & 80 \\
\hline Etiocholanolone-S & 0.81 & 71 & 80 \\
\hline 16OH DHEA-S & 0.74 & 74 & 76 \\
\hline 16/D & 0.84 & 81 & 80 \\
\hline 16/E & 0.85 & 76 & 85 \\
\hline
\end{tabular}

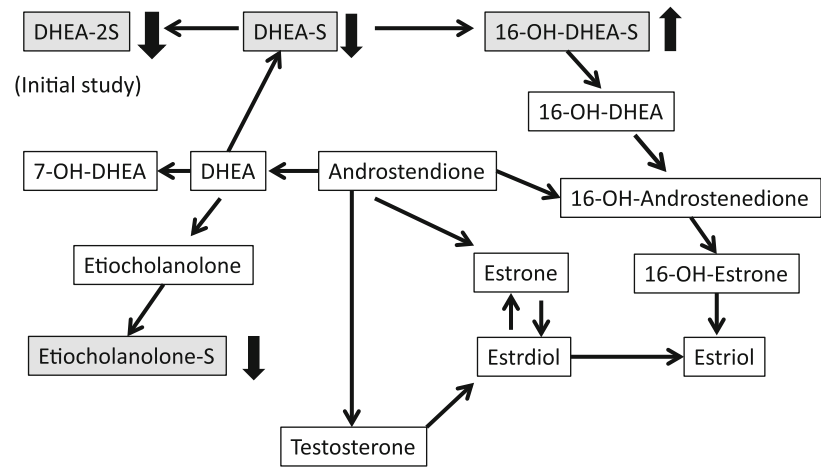

The change was confirmed in this study

Fig. 4 Sex hormone biosynthesis. Grey box shows changes with the progression of fibrosis that were confirmed in this study. Big upward and downward arrows indicated serum levels in advanced fibrosis

increased in patients with breast cancer and endometrial cancer [29], the biological role of this intermediate in liver disease remains unclear. Our validation analysis confirmed that DHEA-S, etiocholanolone-S, and 16-OH-DHEA-S are useful serum markers for detecting fibrosis in NAFLD. In addition, the 16/D and 16/E ratios were even more clearly associated with the grade of fibrosis.

Supplementary Tables $1 \mathrm{~A}$ and B show candidates for biomarkers of inflammation (activity) and steatosis.
Steatosis grade and activity grade were influenced by fibrosis. Therefore, we need further analysis to discover real novel biomarkers for inflammation and steatosis in NAFLD.

We compared these metabolites between simple steatosis and NASH. However, as there were only 8 simple steatosis cases in the initial and validation studies, we could not find novel markers for the discrimination between simple steatosis and NASH.

However, there are limitations to the use of these novel fibrosis markers in NAFLD. When we investigated the influence of age and gender on these markers, we found that DHEA-S and etiocholanolone-S both decreased with increasing age (Fig. 5), although 16-OH-DHEA-S was not influenced by age. Regarding the ratios, the $\mathrm{R}$ values of the 16/D and 16/E ratios were decreased compared with those of DHEA-S and etiocholanolone-S $(R$ values; DHEA-S, $R=0.589$; etiocholanolone-S, $R=0.460 ; 16$-OH DHEA-S, $R=-0.165 ; 16 / \mathrm{D}$ ratio, $R=0.334 ; 16 / \mathrm{E}$ ratio, $R=0.294$ ).

Concerning the influence of gender, we found that DHEA-S, etiocholanolone-S, and the 16/E ratio were significantly different between male and female patients, although 16-OH-DHEA-S and the 16/D ratio showed no influence of gender (Table 3). To investigate the effect of gender and age in our results, we performed 5 multivariate analyses including for each marker, age and gender. In the results, etiocholanolone-S, 16-OH-DHEA-S and both 

(A) DHEA-S
(B) Etiocholanolone-S

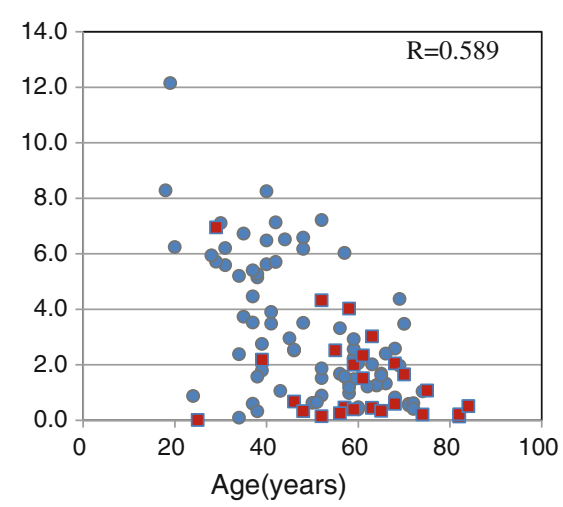

(D) $16 / \mathrm{D}$ ratio
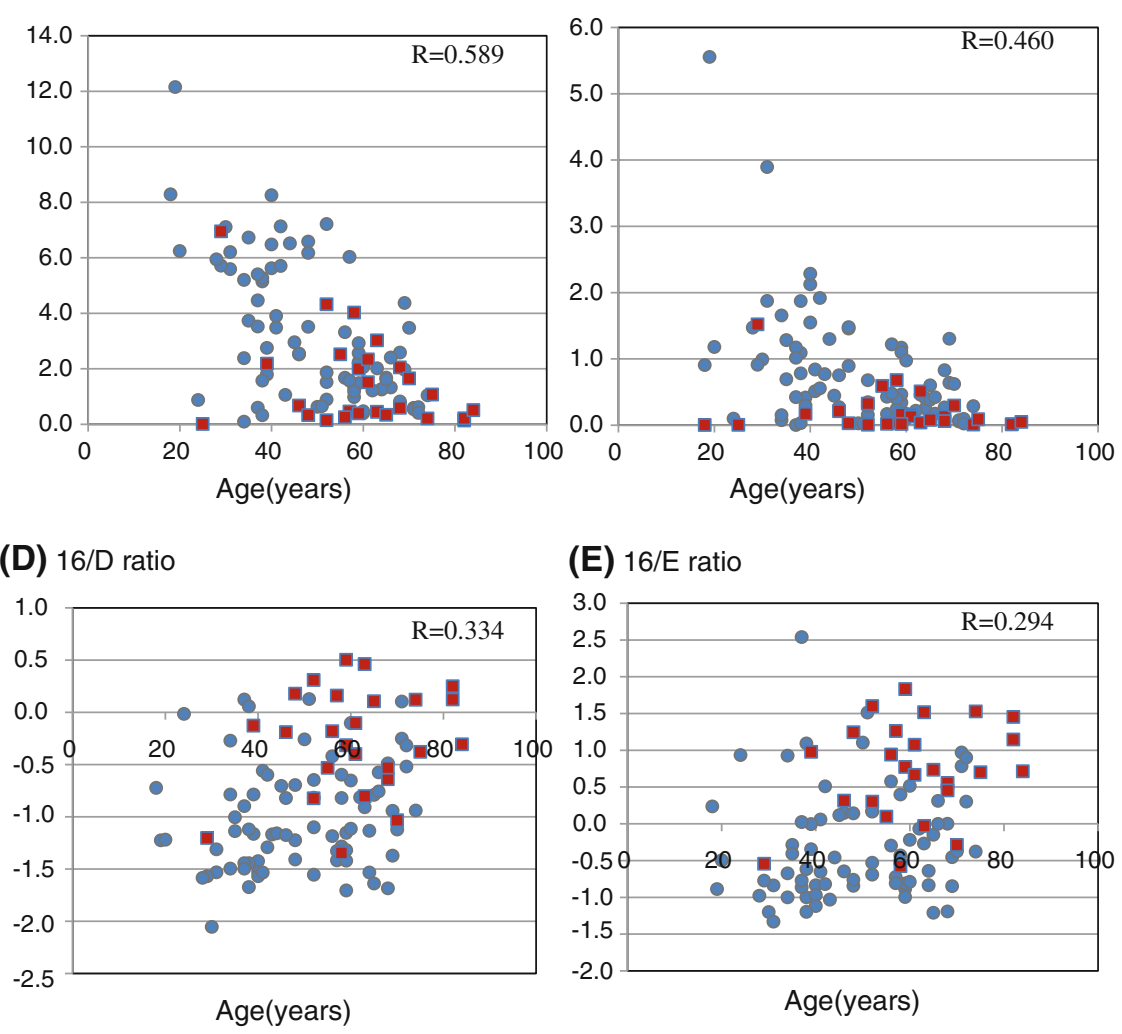

(E) $16 /$ E ratio

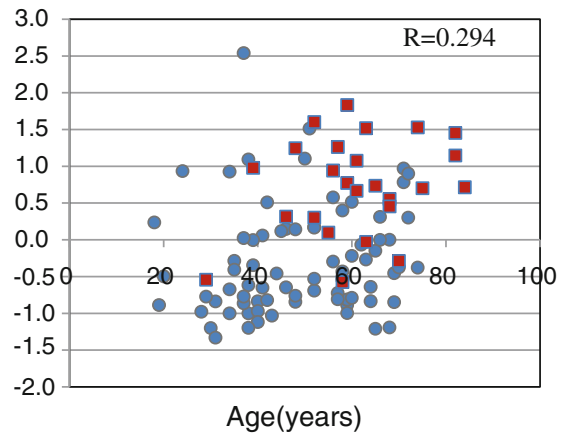

(C) $160 \mathrm{H}$ DHEA-S

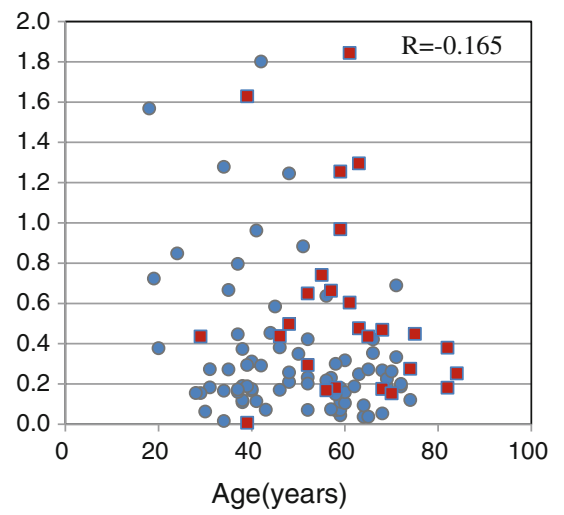

Fig. 5 Association between biomarkers and age. Serum concentrations of DHEA-S (a), etiocholanolone-S (b), 16-OH-DHEA-S (c), and the two ratios (d-e). DHEA-S and etiocholanolone-S decreased with increasing age. 16-OH-DHEA-S was not influenced by age. The $R$ values of the 16/D and 16/E ratios were decreased compared with

Table 3 Gender difference

\begin{tabular}{lrrl}
\hline & \multicolumn{1}{l}{ Female } & \multicolumn{1}{l}{ Male } & $p$ value \\
\hline DHEA-S & $2.085 \pm 2.020$ & $3.400 \pm 2.609$ & 0.0048 \\
Etiocholanolone-S & $0.361 \pm 0.417$ & $0.866 \pm 0.966$ & 0.0006 \\
16OH DHEA-S & $0.342 \pm 0.324$ & $0.442 \pm 0.437$ & N.S. \\
16OH DHEA-S/ & $-0.736 \pm 0.546$ & $-0.893 \pm 0.625$ & N.S. \\
$\quad$ DHEA-S & & & \\
16OH DHEA-S/ & $0.156 \pm 0.835$ & $-0.201 \pm 0.820$ & 0.032 \\
$\quad$ Etiocholanolone-S & & & \\
\hline
\end{tabular}

Mean \pm SD (standard deviation) were expressed

$N S$ not significant

ratios were significant independent markers of comparison between mild fibrosis and severe fibrosis (supplementary Table 2). Both rations only showed small effects of age and gender. Because these compounds are precursors and intermediates of estrogen biosynthesis, their ratios represent the flux of this metabolic pathway. Therefore, our findings suggest that a disturbance of hormone metabolism, which cannot be detected by classical target analysis, is associated with the progression of fibrosis in NAFLD and those of DHEA-S and etiocholanolone-S. $R$ Pearson's correlation coefficient. Blue circles, F0, F1, and F2 NAFLD patients. Red squares, F3 and F4 NAFLD patients. 16/D, 16-OH-DHEA-S/DHEA-S; 16/E, 16-OH-DHEA-S/etiocholanolone-S

that these indicators are useful for assessment of the pathology of NAFLD. Recently, adrenal dysfunction in liver diseases became a target of attention [30]. In addition, Lonardo et al. reported that many endocrine derangements were associated with NAFLD through changes in energy and glycolipid homeostasis and/or a central shift in body fat distribution [31]. Naugler et al. [32] reported that estrogen-mediated inhibition of IL-6 production reduced the liver cancer risk in females. Our data suggest that androgen metabolism might be decreased and estrogen metabolism might be increased. It remains a future issue whether sex hormone metabolism and IL-6 are associated with progression and carcinogenesis in NAFLD. In PBC patients, DHEA-S and etiocholanolone-S were not associated with the stage of fibrosis. Thus, these changes were only seen in NAFLD and may have an important role in its pathogenesis.

By using metabolomic screening, we found some novel biomarkers that suggested disturbance of hormone metabolism is associated with progression of fibrosis in NAFLD. It may be important to investigate the mechanisms of these changes and their pathological significance in NAFLD, 
since the results could lead to the development of new therapies. In conclusion, we hope that our findings contribute to understanding both the clinical and pathological aspects of NAFLD.

Acknowledgments This work was supported in part by a grant-in aid from the Ministry of Health, Labor and Welfare of Japan.

Conflict of interest The authors declare that they have no conflict of interest.

Open Access This article is distributed under the terms of the Creative Commons Attribution Noncommercial License which permits any noncommercial use, distribution, and reproduction in any medium, provided the original author(s) and the source are credited.

\section{References}

1. Teli MR, James OFW, Burt AD, Bennet MK, Day CP. The natural history of nonalcoholic fatty liver: follow-up study. Hepatology. 1995;22:1714-9.

2. Neuschwander-Tetri BA, Caldwell SH. Nonalcoholic steatohepatitis; summary of an AASLD single topic conference. Hepatology. 2003;37:1202-19.

3. Adams LA, Lymp JF, St Sauver J, Sanderson SO, Lindor KD, Feldstein A, et al. The natural history of nonalcoholic fatty liver disease: a population-based cohort study. Gastroenterology. 2005;129:113-21.

4. Hashimoto E, Farrell GC. Will non-invasive markers replace liver biopsy for diagnosing and staging fibrosis in non-alcoholic steatohepatitis? J Gastroenterol Hepatol. 2009;24:501-3.

5. Younossi ZM, Stepanova M, Rafiq N, Makhlouf H, Younoszai Z, Agrawal R, et al. Pathologic criteria for nonalcoholic steatohepatitis: interprotocol agreement and ability to predict liver-related mortality. Hepatology. 2011;53:1874-82.

6. Sreekumar A, Poisson LM, Rajendiran TM, Khan AP, Cao Q, Yu J, Laxman B, et al. Metabolomic profiles delineate potential role for sarcosine in prostate cancer progression. Nature. 2009;457: 910-4.

7. Bogdanov M, Matson WR, Wang L, Matson T, Saunders-Pullman R, Bressman SS, et al. Metabolomic profiling to develop blood biomarkers for Parkinson's disease. Brain. 2008;13: 389-96.

8. Wang C, Kong H, Guan Y, Yang J, Gu J, Yang S, et al. Plasma phospholipid metabolic profiling and biomarkers of type 2 diabetes mellitus based on high-performance liquid chromatography/ electrospray mass spectrometry and multivariate statistical analysis. Anal Chem. 2005;77:4108-16.

9. Sabatine MS, Liu E, Morrow DA, Heller E, McCarroll R, Wiegand R, et al. Metabolomic identification of novel biomarkers of myocardial ischemia. Circulation. 2005;112:3868-75.

10. Heazell AE, Brown M, Worton SA, Dunn WB. Review: the effects of oxygen on normal and pre-eclamptic placental tissue-insights from metabolomics. Placenta. 2011;32(Suppl 2):S119-24.

11. Kalhan SC, Guo L, Edmison J, Dasarathy S, McCullough AJ, Hanson RW, et al. Plasma metabolomic profile in nonalcoholic fatty liver disease. Metabolism. 2011;60:404-13.

12. Puri P, Wiest MM, Cheung O, Mirsahi F, Sargent C, Min HK, et al. The plasma lipidomic signature of nonalcoholic steatohepatitis. Hepatology. 2009;50:1827-38.

13. Barr J, Caballeria J, Martinez-Arranz I, Dominguez-Diez A, Alonso $\mathrm{C}$, Muntane $\mathrm{J}$, et al. Obesity-dependent metabolic signatures associated with nonalcoholic fatty liver disease progression. J Proteome Res. 2012;6:2512-32.

14. Brunt EM, Janney CG, Di Biscrglie AM, Neuschwander-Tetri BA, Bacon BR. Nonalcoholic steatohepatitis: a proposal for grading and staining the histological lesions. Am J Gastroenterol. 1999;94:2467-74.

15. Brunt EM. Nonalcoholic steatohepatitis: definition and pathology. Semin Liver Dis. 2001;21:3-16.

16. Inoue K, Hirohara J, Nakano T, Seki T, Sasaki H, Higuchi K, et al. Prediction of prognosis of primary biliary cirrhosis in Japan. Liver. 1995;15:70-7.

17. Scheuer P. Primary biliary cirrhosis. Proc R Soc Med. 1967;60: 1257-60.

18. Soga T, Igarashi K, Ito C, Mizobuchi K, Zimmermann HP, Tomita M. Metabolomic profiling of anionic metabolites by capillary electrophoresis mass spectrometry. Anal Chem. 2009;81: 6165-74.

19. Awata N, Yamamoto K, Sugimoto I. Absorption, excretion and metabolism of dehydroepiandrosterone sulfate in rabbits. Yakugaku Zasshi. 1978;98:880-5.

20. Kasumov T, Edmison JM, Dasarathy S, Bennett C, Lopez R, Kalhan SC. Plasma levels of asymmetric dimethylarginine in patients with biopsy-proven nonalcoholic fatty liver disease. Metabolism. 2011;60:776-81.

21. Charlton M, Angulo P, Chalasani N, Merriman R, Viker K, Charatcharoenwitthaya $\mathrm{P}$, et al. Low circulating levels of dehydroepiandrosterone in histologically advanced nonalcoholic fatty liver disease. Hepatology. 2008;47:484-92.

22. Bianchi G, Marchesini G, Brunetti N, Manicardi E, Montuschi F, Chianese R, et al. Impaired insulin-mediated amino acid plasma disappearance in non-alcoholic fatty liver disease: a feature of insulin resistance. Dig Liver Dis. 2003;35:722-7.

23. Mong MC, Chao CY, Yin MC. Histidine and carnosine alleviated hepatic steatosis in mice consumed high saturated fat diet. Eur J Pharmacol. 2011;653:82-8.

24. Kwon do Y, Jung YS, Kim SJ, Park HK, Park JH, Kim YC. Impaired sulfur-amino acid metabolism and oxidative stress in nonalcoholic fatty liver are alleviated by betaine supplementation in rats. J Nutr 2009;139:63-8.

25. Nocito A, Dahm F, Jochum W, Jang JH, Georgiev P, Bader M, et al. Serotonin mediates oxidative stress and mitochondrial toxicity in a murine model of nonalcoholic steatohepatitis. Gastroenterology. 2007;133:608-18.

26. Matsuda Y, Fujita T, Hada T, Higashino K. Comparative study on the correlation of plasma gamma-aminobutyric acid and pipecolic acid with liver function in patients with liver cirrhosis. Hepatol Res. 2000;18:132-40.

27. Lasco A, Frisina N, Morabito N, Gaudio A, Morini E, Trifiletti A, et al. Metabolic effects of dehydroepiandrosterone replacement therapy in postmenopausal women. Eur J Endocrinol. 2001;145: 457-61.

28. Hampl R, Starka L. Mini review: 16-a-hydrpxylated metabolites of dehydroepiandrosterone and their biological significance. Endrocrine Reg. 2000;34:161-3.

29. Fishman J, Schneider J, Hershcope RJ, Bradlow HL. Increased estrogen-16 alpha-hydroxylase activity in women with breast and endometrial cancer. J Steroid Biochem. 1984;20:1077-81.

30. Fede G, Spadaro L, Tomaselli T, Privitera G, Germani G, Tsochatzis E, et al. Adrenocortical dysfunction in liver disease: a systematic review. Hepatology. 2012;55:1282-91.

31. Lonardo A, Carani C, Carulli N, Loria P. 'Endocrine NAFLD' a hormonocentric perspective of nonalcoholic fatty liver disease pathogenesis. J Hepatol. 2006;44:1196-207.

32. Naugler WE, Sakurai T, Kim S, Maeda S, Kim K, Elsharkawy $\mathrm{AM}$, et al. Gender disparity in liver cancer due to sex differences in MyD88-dependent IL-6 production. Science. 2007;317:121-4. 